\title{
Research on Supermarket Customer Classification Based on Agent Ant Colony Algorithm
}

\author{
Hong Xue, Wenchao Lu, Peiyuan Guo \\ College of Computer and Information Engineering \\ Beijing Technology and Business University \\ Beijing, China \\ e-mail: hongxue6@yahoo.com.cn
}

\begin{abstract}
The Ant colony algorithm is an intelligent algorithm with its better robustness and parallelism, as well as the advantage combined easily with other algorithms. Each ant Agent does not need to have a comprehensive understanding to the every aspect of the system so the individual Agent is considered as the main object of study. In the paper, supermarket customer subdivision model was researched by the self-organizational peculiarity of ant colony algorithm and the intelligent advantage of Agent, which not only embodied the principle of simplicity but also achieved better customer subdivision results. Considering the peculiarities of supermarket customers, ant colony algorithm was combined with Agent, which was applied to research of supermarket customer subdivision model. Adopting the Repast J simulation platform, the process and results of simulation were more intuitive. The results show that the algorithm has the better validity and stability and can provide more effective support for decision-making of governor in the area of supermarket customer management.
\end{abstract}

Keywords-Agent; Ant Colony Algorithm; Chain Supermarket; Customer Classification

\section{INTRODUCTION}

With the popularization and expansion of supermarket in every area, there are more chain supermarkets than before. With the increasingly fierce competition, marketing models centered in customers have become more and more important in customer relation management. Facing the massive data collected from POS system every day, how to use these data effectively has become the problem that needs urgently to solve and has also become a very valuable research.

Customer classification was one of the most important parts in supermarket customer relation management. The different marketing strategies were applied to the classified customers, which was the key to determine the success or failure of the supermarket management. Traditional customer classification methods are mostly based on the experience of decisionmakers or the methods dividing simple property characters of customers, which is very difficult to meet requirement of further analysis for customers. With the massive customer data, the traditional methods appear to be incompetent. The data mining technology becomes a research hotspot in this field [1]. This paper put forward a new ant colony data mining algorithm based on Agent, which was applied to the study of supermarket customer classification.
Nowadays, there are many research results about data classification and ant colony algorithm. But the application of ant colony algorithm in the classification problem is few. That ant colony algorithm combined with Agent is applied to classification problem is a new research direction. As for mass data of supermarket, it was difficult to grasp clearly the relationship between them. This paper combined ant colony algorithm with Agent. The mechanism character of selforganizing allowed that each individual ant Agent did not understand comprehensively every aspect of the system. The ant colony algorithm was an intelligent algorithm with its better robustness and parallelism, as well as the advantage combined easily with other algorithms, which made the subdivision model have better classification effect.

\section{CluSTERING ANALYSIS OF SUPERMARKET CUSTOMERS BASED ON ANT COLONY ALGORITHM}

Clustering analysis methods include K-means algorithm, SOM neural network algorithm, hierarchical algorithm, fuzzy C-means algorithm, and so on. The ant colony algorithm applied to clustering analysis is based on the behaviors of ant heaping dead bodies and classing children. Deneubourg J L established a basic model based on phenomenon of ant colony clustering. Lumer E and Faieta B applied the model to the areas of data analysis [2], which was established based mainly on the behavior manner of single ant picking up and putting down objects. First of all, the initial clustering data was randomly distributed on a two-dimensional plane. Then a number of fictitious ants distributed on the plane moved randomly and decided whether to pick up or to put down their objects to achieve the purpose of clustering according to the rules.

In this paper, the logical construction of supermarket customer classification based on ant colony algorithm is shown in Fig. 1.

The entire classification system standardized the expression of combinatorial optimization problem to form a standard ant colony movement planning. Then each individual ant's information was real time updated according to specific rules. The whole colony was managed through updating management system. Finally, the task of the entire system was realized by

\footnotetext{
* This paper was supported by the Education Committee

Foundation of Beijing (200809) and (KM200610011006).
} 


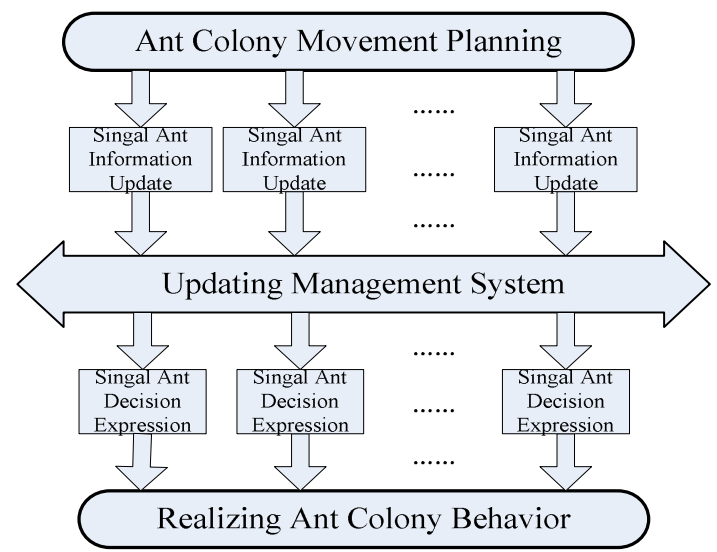

Figure 1. Logical struction figure of supermarket customer classification based on ant colony algorithm

the behaviors after each individual made decision and was updated. Compared with the traditional classification algorithm, the self-organizing property of ant colony algorithm has been improved greatly when ant colony algorithm was applied to the supermarket customer classification system. A number of traditional classification algorithms are specific solutions for specific issues, which must be based on a clear understanding to the issues. So it's difficult to solve different problems. However the ant colony algorithm was not subjected to these restrictions and can be applied easily to a kind of problem [3].

\section{ANT COLONY ALGORITHM OF SUPERMARKET CUSTOMER CLASSIFICATION BASED ON AGENT}

This paper combined the self-organizational property of ant colony algorithm with the intelligent advantage of Agent. The individual Agent was considered as the main object of study. The supermarket customer subdivision model was researched by the ant colony algorithm combined with Agent, which not only embodied the principle of simplicity but also achieved better customer subdivision results [4]. Specific flow of the ant colony algorithm of supermarket customer classification based on Agent is as follow:

1) Parameters initialization. The data objects of supermarket customers and the ant number of ant colony are initialized.

2) The data objects of supermarket customers would be randomly distributed to a two-dimensional plane, which would allocate a pair of coordinate values $(x, y)$ to each data object.

3) The fictitious ants of ant colony are defined as Agents. They are randomly distributed to same plane and choose a data object, which makes each ant initialized as loading.

4) The ant Agents move randomly on the space. If the whole system meets the finish condition, the program would go to 13) step, otherwise it continues to carry out 5) step.

5) If there is an ant Agent at the anticipated position, the program returns to 4) step, otherwise it would continue to carry out 6) step to avoid collision.

6) If the Agent has been not loaded, the program jumps to 10) step, otherwise it would continue to carry out 7) step.
7) The similarity between load of Agent and the surrounding data is calculated.

8) If the similarity has reached the requirement of classification, the program would continue to carry out 9) step, otherwise it jumps to 4) step.

9) If there is no the data object on the current position, the object is put down and Agent is marked as empty state. Then the program would jump to 4) step.

10) If there is no the data object on the current position, the program would jump to 4) step, otherwise it continues to carry out 11) step.

11) The similarity between the data object on the current position and the surrounding object is calculated. If the similarity has reached the requirement of classification, the program would continue to carry out 12) step, otherwise it jumps to 4) step.

12) Ant Agent picks up the object and marks itself as loading. Then the program would jump to 4) step.

13) The all customers on the plane have reached the classification requirement. The program ends.

The flow chart of customer subdivision ant colony algorithm based on Agent is shown in Fig.2.

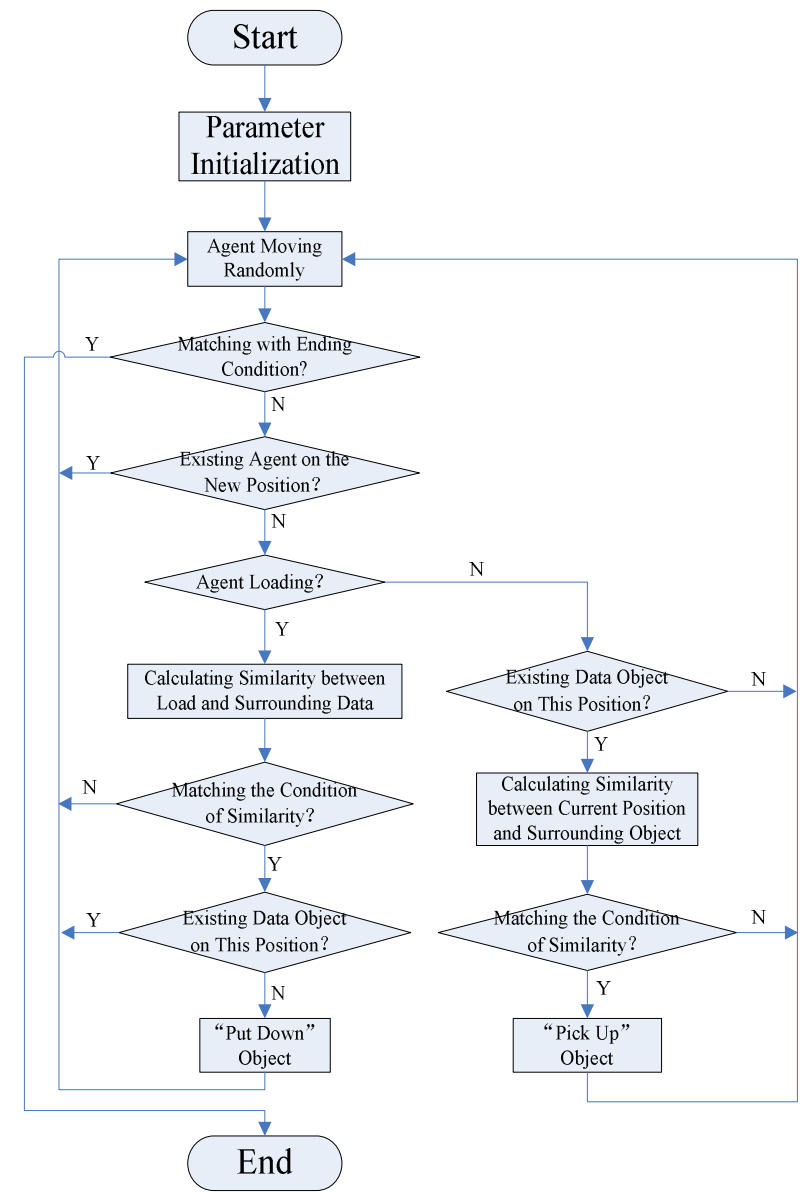

Figure 2. Flow chart of customer subdivision ant colony algorithm based on Agent 


\section{SIMULATION RESEARCH ON CHAIN SUPERMARKET CUSTOMER CLASSIFICATION}

The multi-agent modeling tool, Repast, adopts OO (Object Oriented) design method and provides a favorable programming framework. The simulation procedure was divided into two large modules. The core classes included the Agent class, Model class, Action class, and DataSource class. The optional classes included Space class, SimulationDataVisualization class and some assistant function classes. Agent class defined the property and behavior of Agent. Model class was the core of Repast simulation program. A simulation program has one Model class. Action class was the decoupling between simulation scheduling and Agent class. DataSource class played a decoupling part between AnalysisDisplay class and Agent class of simulation program [5].

The simulation program included three classes:

1) AntModel class. This class realized the preparatory phase of simulation and practical operation, which was the core class of the whole program, and established the program framework of SimModelImpl abstract class.

2) AntAgent class. This class defined the property and behavior of Agent. Because Agents in simulation model were coessential, an Agent class was needed.

3) AntSpace class. This class realized the activity space of Agent and data objects and defined the behavior of Agent in the space.

Specific UML class figure is shown in Fig. 3.

In Fig.3, three classes completed different tasks by cooperation. The relationship of AntModel class, AntAgent class and AntSpace class was a depended relationship, which is showed in Fig. 4.

The function realization of AntModel class needed the support of AntAgent class and AntSpace class, which was the property of depended relationship and implied that the AntModel class would be incomplete without support of the other two classes. AntModel class used the instantiation objects of the other two classes as its parameters.

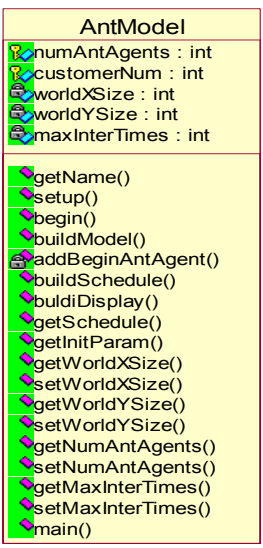

\begin{tabular}{|c|}
\hline 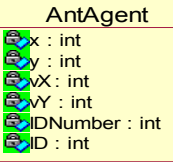 \\
\hline 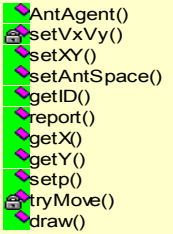 \\
\hline
\end{tabular}

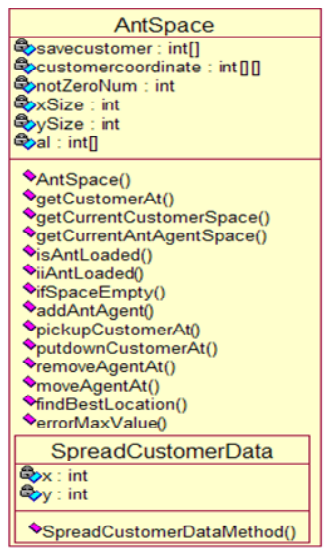

Figure 3. Figure of primary program classes

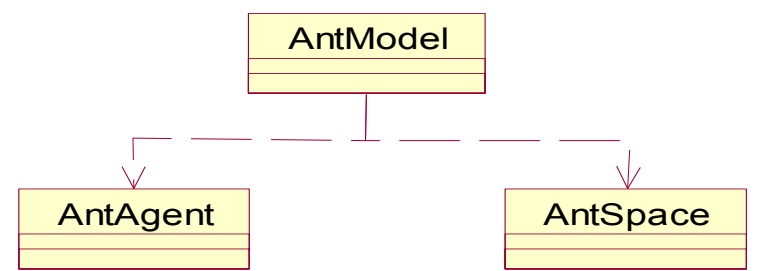

Figure 4. Relationship among classes

After the program was initialized, the data objects that represented supermarket customers and the fictitious ant Agents were distributed on the two-dimensional plane, which is showed in Fig. 5. This paper selected 300 supermarket customers as the data objects to research customer classification. The number of Agent selected was 10 .

In Fig. 5, red represented supermarket customer. Blue represented Agent. Here each Agent was initialized as loading. Agent was randomly distributed on the place at where there was customer. Agent picked up the customer on this position.

After the program ran, Agent moved freely on the plane. It will decide whether to put down or to pick up customer according to the rules. When the results of program running reached the requirement of classification, Agent will more slow move, which meant that the results of classification were basically stable. The results of classification are shown in Fig.6.

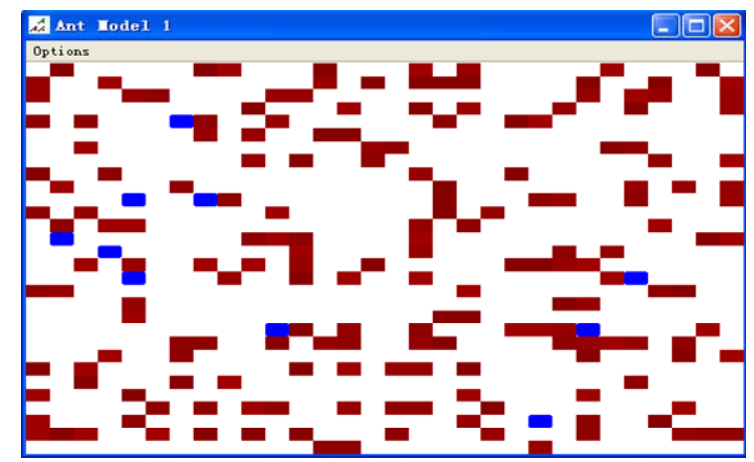

Figure 5. The data objects and Agents distributed on the two-dimension plane

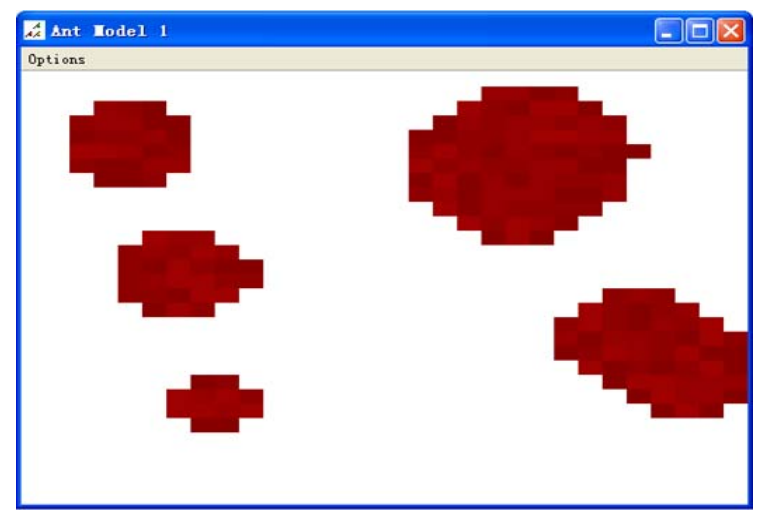

Figure 6. The results of classification 
In Fig. 6, Agents divided 300 customers into 5 types. The simulation results met the requirement of system. That ant colony algorithm was combined with Agent was successfully applied to research of supermarket customer subdivision model. Supermarket governors could allocate five kinds of member cards, such as diamond card, golden card, silver card, copper card and the general card, to customers according to the results of classification. Then they could take corresponding marketing strategies according to the characters of these customers. In application, according to the specific situation of each supermarket, the properties of classification are selected and classification is carried out based on RFM index. The different manners of sales promotion, such as advertising sales promotion, discounting sales promotion, member card merchandise sales promotion, e-mail sales promotion, could be adopted to different kinds of customers in order to achieving a win-win purpose between supermarket and customers [6].

\section{CONCLUSION}

Ant colony algorithm is a new bionic evolvement algorithm. Although genetic algorithm, artificial neural nets, artificial immune algorithm, artificial shoal algorithm and ant colony algorithm belong to a kind of bionic optimizing algorithm that simulates biology system in nature, the positive feedback mechanism of ant colony algorithm is a marked peculiarity compared with other algorithms. It is an essential parallel algorithm, which will greatly reduce the computing time. In this paper, the ant colony algorithm combined with
Agent was applied to research of supermarket customer subdivision model and each ant was regarded as one Agent, which not only embodied the strong ability of solving problem and robustness of ant colony algorithm for classification but also embodied freedom ability and fast reaction ability of Agent. Better customer subdivision results were achieved. The simulation results show that the ant colony algorithm based on Agent is more intelligent compared to the traditional classification algorithms and the process and results of classification are more intuitive by adopting the Repast $\mathrm{J}$ simulation platform.

\section{REFERENCES}

[1] Zhang Zhiqin, A Customer Segmentation Research Based on Data Mining [D], Guangzhou: Jinan University, 2007.

[2] Tang Yijun, Application Research on Data Ming Using Ant Colony Algorithm [D], Shenyang: Liaoning Technical University, 2007.

[3] Lumer E, Faieta B, Diversity and adaptation in populations of clustering ants [J], Proceedings of the 3rd international conference on simulation of adaptive behavior:from animals to animals, 1994, pp.499508.

[4] Matthia Kluseh, Information Agent technology for the Internet: A survey[J], Data \& Knowledge Engineering, 2001, 36:pp.337-372.

[5] Liao Shouyi, Research on Methodology of Agent-Based Modeling and Simulation for Complex Systems and Application [D], Changsha: National University of Defense Technology, 2005.

[6] Duan Haibing, Ant Colony Algorithm Theory and Its Application [M], Science Press, Beijing, China, 2005. 